\title{
Preventing masks from becoming the next plastic problem
}

\author{
Elvis Genbo Xu $(\bowtie){ }^{1}$, Zhiyong Jason Ren $(\bowtie)^{2}$ \\ 1 Department of Biology, University of Southern Denmark, Odense 5230, Denmark \\ 2 Department of Civil and Environmental Engineering and the Andlinger Center for Energy and the Environment, \\ Princeton University, Princeton, NJ 08544, USA
}

(C) Higher Education Press 2021

Face masks help prevent the spread of coronavirus and other diseases, and mass masking is recommended by almost all health groups and countries to control the COVID-19 pandemic (Brooks et al., 2020). Recent studies estimated an astounding 129 billion face masks being used globally every month ( 3 million / minute) and most are disposable face masks made from plastic microfibers (Prata et al., 2020). With increasing reports on inappropriate disposal of masks, it is urgent to recognize this potential environmental threat and prevent it from becoming the next plastic problem.

Waste plastics are one of the most prevalent environmental pollutants today. Even before COVID, over 300 million tons of plastics are produced globally per year and most end up in nature as waste (Geyer et al., 2017). Plastic products can not be readily biodegraded but fragment into smaller plastic particles, namely micro- and nanoplastics that widespread in ecosystems (Alimi et al., 2018; Nguyen et al., 2019). Ingestion of microplastics is known to cause direct adverse effects and also expose organisms to toxic chemicals and pathogenic microorganisms (Vethaak and Leslie, 2016).

The pandemic and use of masks: Similar to the "throwaway living" style for other plastic products, disposable masks have symbolized pandemics from 2003 SARS to COVID-19 (Syed et al., 2003; Elachola et al., 2020). Although there is no official report on how many masks are disposed of, studies estimated billions of masks are needed monthly. China as the largest mask producer increased its production by a factor of 10 in March 2020 (source: "The daily output of masks exceeding 100 million" on Xinhua Daily Telegraph, 3 March 2020). Globally, a recent study estimated a monthly use of 129 billion face masks (Prata et al., 2020). This puts disposable masks on a similar scale as plastic bottles, which is

Received November 18, 2020; Revised January 26, 2021; Accepted January 27, 2021; Available online February 28, 2021

E-mails: elvis@biology.sdu.dk (E. Xu), zjren@princeton.edu (Z. Ren) estimated to be 43 billion per month. However, different from plastic bottles, $\sim 25 \%$ of which is recycled, there is no official guidance on mask recycle, making it more likely to be disposed of as solid waste (Fadare and Okoffo, 2020).

Mask materials and environmental fates and impacts: The common disposable surgical masks are made of three layers. The outer layer is made up of nonabsorbent material (e.g., polyester) that protects against liquid splashes. The middle layer is non-woven fabrics (e. g., polypropylene and polystyrene) created using a meltblowing process, which prevents droplets and aerosols via an electrostatic effect. The inner layer is made of absorbent material like cotton to absorb vapor (Fig. 1). Different polymers are used in mask manufacturing, and fabric polypropylene is used the most. Polypropylene is one of the most commonly produced plastics and the high usage has led to a large waste accumulation in the environment (Andrady, 2011). Once in the environment, the mask is subjected to solar radiation and heat, but the degradation of polypropylene is retarded due to its high hydrophobicity, high molecular weight, lacking an active functional group, and continuous chain of repetitive methylene units. These recalcitrant properties lead to the persistence and accumulation in the environment. The in situ weathering can generate a large number of micro-sized polypropylene particles $(<5 \mathrm{~mm}$ ) during a relatively short period (weeks) and further fragment into nanoplastics $(<1 \mu \mathrm{m})$ (Mattsson et al., 2018).

When not properly collected and managed, masks can be transported from land into freshwater and marine environments by surface run-off, river flows, oceanic currents, wind, and animals (via entanglement or ingestion) (Fig. 1). The occurrence of waste masks has been increasingly reported in different environments and social media have shared of wildlife tangled in elastic straps of masks. The author also observes disposable surgical masks in Odense, Denmark (Fig. 2). Like other plastic debris, disposable masks may accumulate and release harmful chemical and biological substances, such as bisphenol A, heavy metals, as well as pathogenic micro-organisms. Moreover, the 


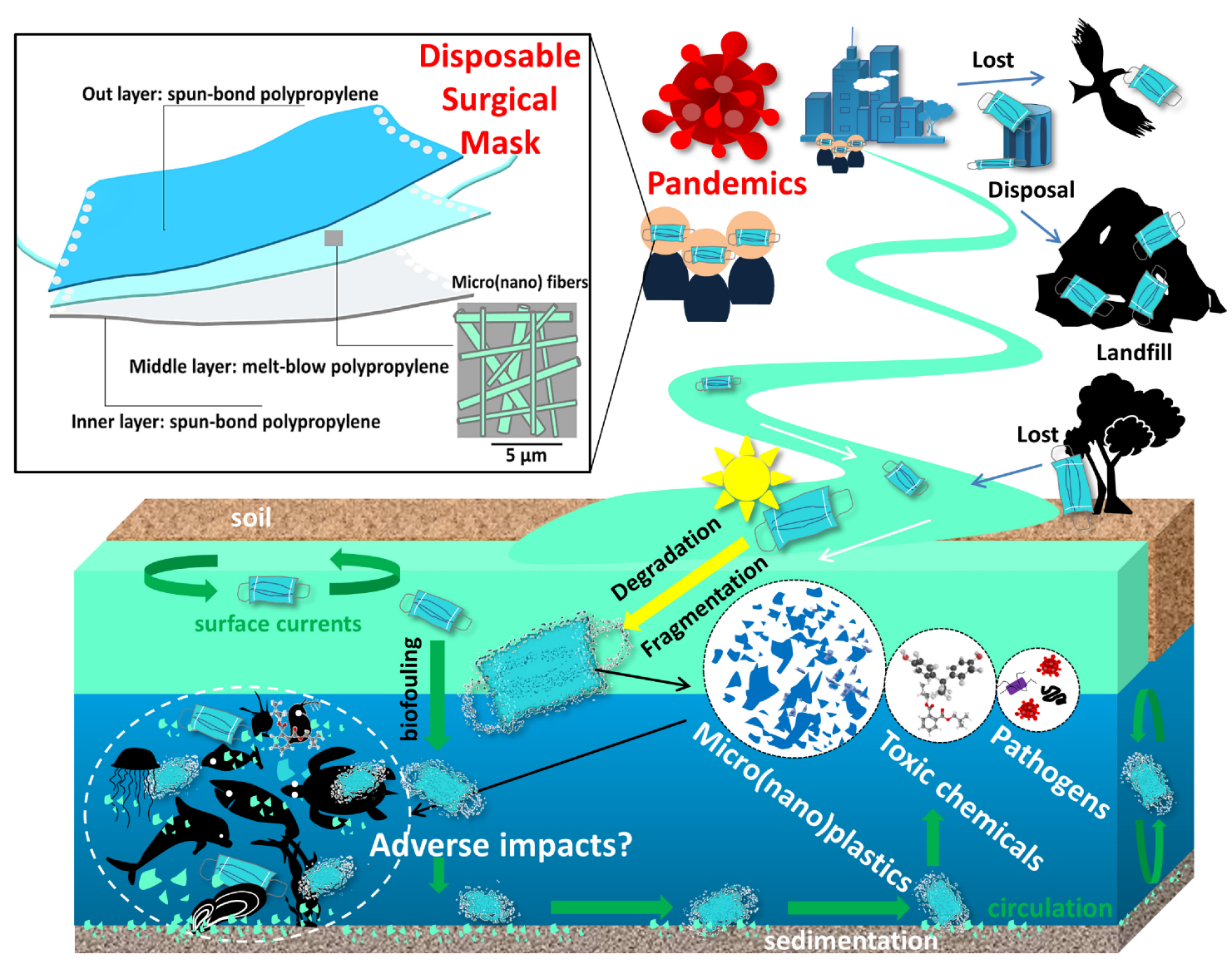

Fig. 1 The potential environmental fates and impacts of disposable surgical masks.

uptake of small plastic particles is known to cause adverse health effects by three main possible means: particle toxicity, chemical toxicity, and pathogenic microorganism vectors (Vethaak and Leslie, 2016). A newer and bigger concern is that the masks are directly made from microsized plastic fibers (thickness of $\sim 1$ to $10 \mu \mathrm{m}$ ). When breaking down in the environment, the mask may release more micro-sized plastics, easier and faster than bulk plastics like plastic bags. Such impacts can be worsened by a new-generation mask, nanomasks, which directly use nano-sized plastic fibers (e.g., diameter $<1 \mu \mathrm{m}$ ) and add a new source of nanoplastic pollution. However, no data on mask degradation in nature exists, so we simply do not know how masks contribute to the large number of plastic particles detected in the environment.

Best practices and research needs: Disposable plastic items including masks are irreplaceable in fighting the pandemic, and the concerns about reusable plastics as vectors for virus have led to delays in recycling programs and single-use plastic regulations. While there are concerns about transmission via contaminated household plastic items, the probability of such transmission is considered low compared to personal protection equipment (PPE) residues that are more likely in contact with the virus. Rather the dramatic increase in disposal of such single-use plastics poses a potential threat to the environment. Such impacts in global plastic pollution are essentially unknown. Thus, the environmental research community needs to move fast to understand and mitigate these risks. Critical rethinking of the three 'Rs' can be valuable: regulate (life-cycle evaluation on production, disposal, and decontamination), reuse (washable masks), and replace (biodegradable materials) single-use plastic masks. In this effort, interdisciplinary research is urgently needed on the environmental fates of disposable masks, including transportation, accumulation, fragmentation, degradation, release of micro- and nanoplastics, harmful chemicals and pathogens, and potential effects on life. Programs can be made to set up mask-only trash cans for collection and disposal. Standardization, guidelines, and strict implementation of waste management for mask wastes should be considered (Sangkham, 2020). Reusable face masks like cotton masks are recommended to replace disposable masks. Other development can be made to manufacture biodegradable disposal masks but the higher cost and unknown safety of new materials need to be considered. Preventive measures including mask-wearing, vaccinedeveloping, and good hygiene shall all remain. Identification and elimination of the major inputs of mask waste to reduce the likelihood of masks entering the environment 

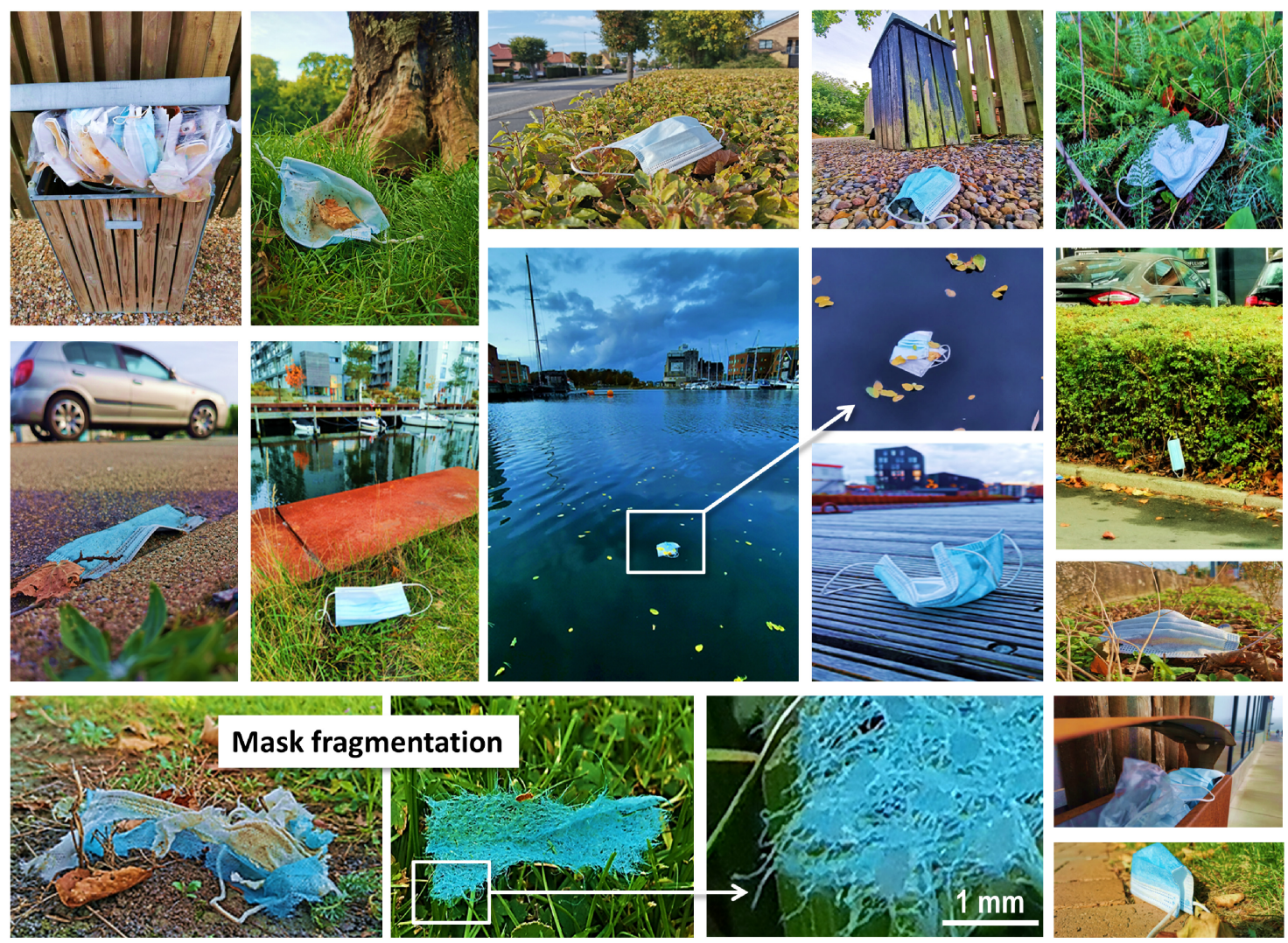

Fig. 2 Disposable surgical masks and mask fragments observed in Odense, Denmark. Photo credit: Dr. Elvis Genbo Xu.

are urgent. "The coronavirus may never go away but just become another endemic virus in our communities", the WHO warns (source: "Coronavirus may never go away, World Health Organization warns" on BBC News, 14 May 2020). It is imperative to launch coordinated efforts from environmental scientists, medical agencies, and solid waste managing organizations, and the general public to minimize the negative impacts of disposal mask, and eventually prevent it from becoming another too-big-tohandle problem.

\section{References}

Alimi O S, Farner Budarz J, Hernandez L M, Tufenkji N (2018). Microplastics and nanoplastics in aquatic environments: Aggregation, deposition, and enhanced contaminant transport. Environmental Science \& Technology, 52(4): 1704-1724

Andrady A L (2011). Microplastics in the marine environment. Marine Pollution Bulletin, 62(8): 1596-1605

Brooks J T, Butler J C, Redfield R R (2020). Universal masking to prevent SARS-CoV-2 transmission - the time is now. Journal of the American Medical Association, 324(7): 635-637

Elachola H, Ebrahim S H, Gozzer E (2020). COVID-19: Facemask use prevalence in international airports in Asia, Europe and the Americas,
March 2020. Travel Medicine and Infectious Disease, 35: 101637

Fadare O O, Okoffo E D (2020). Covid-19 face masks: A potential source of microplastic fibers in the environment. Science of the Total Environment, 737: 140279

Geyer R, Jambeck J R, Law K L (2017). Production, use, and fate of all plastics ever made. Science Advances, 3(7): e1700782

Mattsson K, Jocic S, Doverbratt I, Hansson L A (2018). Nanoplastics in the aquatic environment. In: Zeng E Y, ed. Microplastic Contamination in Aquatic Environments. Amsterdam: Elsevier, 379-399

Nguyen B, Claveau-Mallet D, Hernandez L M, Xu E G, Farner J M, Tufenkji N (2019). Separation and analysis of microplastics and nanoplastics in complex environmental samples. Accounts of Chemical Research, 52(4): 858-866

Prata J C, Silva A L, Walker T R, Duarte A C, Rocha-Santos T (2020). COVID-19 pandemic repercussions on the use and management of plastics. Environmental Science \& Technology, 54(13): 7760-7765

Sangkham S (2020). Face mask and medical waste disposal during the novel COVID-19 pandemic in Asia. Case Studies in Chemical and Environmental Engineering, 2: 100052

Syed Q, Sopwith W, Regan M, Bellis M A (2003). Behind the mask. Journey through an epidemic: Some observations of contrasting public health responses to SARS. Journal of Epidemiology and Community Health, 57(11): 855-856

Vethaak A D, Leslie H A (2016). Plastic debris is a human health issue. Environmental Science \& Technology, 50(13): 6825-6826 